\title{
LUT
}

University

\section{Identification of Lone-Pair Surface States on Indium Oxide}

Davies Daniel W., Walsh Aron, Mudd James J., McConville Chris F., Regoutz Anna, Kahk J. Matthias, Payne David J., Dhanak Vin R., Hesp David, Pussi Katariina, Lee Tien-Lin, Egdell Russell G., Zhang Kelvin H. L.

This is a Post-print version of a publication

published by American Chemical Society

in Journal of Physical Chemistry C

DOI: $10.1021 /$ acs.jpcc.8b08623

Copyright of the original publication: () 2018 American Chemical Society

Please cite the publication as follows:

Davies D. W., Walsh A., Mudd J. J., McConville C. F., Regoutz A., Kahk J. M., Payne D. J., Dhanak V. R., Hesp D., Pussi K., Lee T., Egdell R. G., Zhang K. H. L. (2019). Identification of Lone-Pair Surface States on Indium Oxide. Journal of Physical Chemistry C, vol. 123, 3. pp. 1700-1709. DOI: $10.1021 /$ acs.jpcc.8b08623

This is a parallel published version of an original publication. This version can differ from the original published article. 


\title{
Identification of lone pair surface states on indium oxide
}

\author{
Daniel W Davies, ${ }^{\dagger}$ Aron Walsh, ${ }^{\ddagger}$ James J. Mudd, "Chris F. McConville, ${ }^{\S}, \|$ Anna
} Regoutz, ${ }^{* \ddagger}$ J. Matthias Kahk, ${ }^{\ddagger}$ David J. Payne, ${ }^{\ddagger}$ Vin R. Dhanak, ${ }^{\perp}$ David Hesp,,$^{\perp}$ Katariina Pussi,\# Tien-Lin Lee, ${ }^{\Uparrow}$ Russell G. Egdell, ${ }^{@}$ and Kelvin H. L. Zhang*, $\dagger$ Centre for Sustainable Chemical Technologies, Department of Chemistry, University of Bath, Bath, BA2 7AY, United Kingdom

$\ddagger$ Department of Materials, Imperial College London, London, SW7 2AZ, United Kingdom

IDiamond Light Source Ltd., Didcot, Oxfordshire, OX11 ODE, United Kingdom $\S$ Department of Physics, University of Warwick, Coventry, CV4 7AL, United Kingdom IISchool of Science, RMIT University, Melbourne, Victoria 3001, Australia $\perp$ Department of Physics, University of Liverpool, Liverpool, L69 3BX, United Kingdom \#LUT School of Engineering Science, Lappeenranta University of Technology, P.O. Box 20, FIN-53851 Lappeenranta, Finland

@ Department of Chemistry, University of Oxford, Inorganic Chemistry Laboratory, South Parks Road, Oxford, OX1 3QR, United Kingdom

$\triangle$ State Key Laboratory of Physical Chemistry of Solid Surfaces, College of Chemistry and Chemical Engineering, Xiamen University, Xiamen 361005, Peoples Republic of China

E-mail: a.regoutz@imperial.ac.uk; kelvinzhang@xmu.edu.cn

\begin{abstract}
Indium oxide is widely used as a transparent electrode in optoelectronic devices and as a photocatalyst with activity for the reduction of $\mathrm{CO}_{2}$. However, very little is known
\end{abstract}


about its surface structure. In this report, directional lone-pair surface states due to filled $5 s^{2}$ orbitals have been identified on $\mathrm{In}_{2} \mathrm{O}_{3}(111)$ through a combination of hard and soft X-ray photoemission spectroscopy and density functional theory calculations. The lone pairs reside on indium ad-atoms in a formal +1 oxidation state, each of which traps two electrons into a localised hybrid orbital protruding away from the surface and lying just above the valence band maximum in photoemission spectra. The third electron associated with the ad-atoms is delocalised into the conduction band, thus producing the surface electron accumulation layer identified previously on vacuumannealed $\mathrm{In}_{2} \mathrm{O}_{3}(111)(1 \times 1)$ surfaces. The surface structure is further supported by low-energy electron diffraction. The $5 s^{2}$ lone pairs confer Lewis basicity on the surface In sites, and may have a pronounced impact for the (photo-)catalytic activity of reduced $\mathrm{In}_{2} \mathrm{O}_{3}$.

\section{Introduction}

Metallic elements occupying positions beyond the end of the transition series in the periodic table display two main oxidation states: the group oxidation state $\mathrm{N}$ and the N-2 state. $^{1,2}$ Lower valent N-2 compounds frequently adopt structures in the solid state where the N-2 cations occupy sites with coordination geometries lacking inversion symmetry: $\mathrm{Sn}(\mathrm{II}) \mathrm{O},{ }^{3}$ $\mathrm{Sn}(\mathrm{II}) \mathrm{S},{ }^{4} \mathrm{~Pb}(\mathrm{II}) \mathrm{O},{ }^{5}$ and $\mathrm{Bi}(\mathrm{III})_{2} \mathrm{O}_{3}{ }^{6}$ are just some typical examples. Free ions in the $\mathrm{N}-2$ state such as $\mathrm{Sn}^{2+}$ have an $\mathrm{n} s^{2}$ electron configuration, with empty n $p$ levels. The conventional explanation of the propensity for asymmetric cation sites involves the idea of directional electron lone pairs formed by on-site hybridisation between occupied metal ns states and one of the empty np states, thus lowering the internal electronic energy of the cation through a second-order Jahn-Teller effect. ${ }^{1,2}$ This mixing is not possible at sites with inversion symmetry because $s$ and $p$ states are of opposite parity. However, the metal n $s$ states are generally

much too low in energy to allow direct interaction with metal $\mathrm{n} p$ states. ${ }^{7,8}$ Through a combination of first-principles calculations and X-ray spectroscopy techniques it has been shown 
recently that the $\mathrm{n} s-\mathrm{n} p$ interaction must instead be mediated by anion states, whose energy lies between that of the $\mathrm{n} s$ and $\mathrm{n} p$ states. ${ }^{9,10}$ Nonetheless, the idea of directional lone pair hybrid states as the drivers of the structural distortions remains valid.

Cations occupying sites at a surface cannot experience full inversion symmetry. Moreover, oxides such as $\mathrm{Sn}(\mathrm{IV}) \mathrm{O}_{2}$ and $\mathrm{In}(\mathrm{III})_{2} \mathrm{O}_{3}$ have a propensity toward electron-rich n-type behaviour, with the facile formation of oxygen vacancies. Bulk oxygen vacancies are two electron donors, although whether this donor is deep or shallow is a matter of ongoing debate. ${ }^{11,12}$ At a surface it may be envisaged that the electrons could be trapped in a directional lone pair state protruding out into the vacuum from a surface cation adjacent to an oxygen vacancy. ${ }^{13}$ Lone pair surface states of this sort were first invoked to account for structure in the bandgap of photoemission spectra of $\mathrm{Sn}(\mathrm{IV}) \mathrm{O}_{2} \cdot{ }^{14,15}$ More recently, $\mathrm{Ga}(\mathrm{I})$ lone pairs have been used to rationalise structure in photoemission spectra of ion-bombarded $\mathrm{Ga}(\mathrm{III})_{2} \mathrm{O}_{3} \cdot{ }^{16}$ Lone pair surface states have also been invoked to account for the photocatalytic reduction of $\mathrm{CO}$ on $\mathrm{In}_{2} \mathrm{O}_{3}$ powders. ${ }^{13}$ Nonetheless, unambiguous identification of a lone-pair state on a structurally well-defined oxide surface has remained elusive.

Here an electronic state in the bulk bandgap of vacuum annealed $\operatorname{In}_{2} \mathrm{O}_{3}(111)(1 \times 1)$ is characterised by combined soft and hard X-ray photoemission measurements. Indium oxide adopts the bixbyite structure, based on a $2 \times 2 \times 2$ supercell of the fluorite $\left(\mathrm{CaF}_{2}\right)$ structure with an ordered arrangement of $25 \%$ of the anion sites which are empty. As with fluorite, the (111) surface is the most stable amongst the low index terminations and is based on repeating quadrupolar units with stoichiometry $\left\{\mathrm{O}_{12} \mathrm{In}_{16} \mathrm{O}_{12}\right\}$ and a single dangling bond for each atom at the surface. ${ }^{17}$ Previous analysis of intensity-voltage (I/V) curves in low energy electron diffraction (LEED) for $\operatorname{In}_{2} \mathrm{O}_{3}(111)(1 \times 1)$ suggested an essentially bulk-truncated structure with minor relaxations. ${ }^{18}$ However, more recent work using scanning tunnelling microscopy (STM) has shown that there are in fact two distinct (111) structures: the bulk- 
truncated structure invoked in the previous LEED I/V study; and a new structure containing a single indium ad-atom within each surface unit cell. The latter is formed by annealing under ultrahigh vacuum (UHV), ${ }^{19}$ or by deposition of Fe. ${ }^{20}$ The signature of the ad-atom reconstruction, a hexagonal array of bright spots in STM, was found in our own work using surface preparation procedures similar to those in the LEED experiments. ${ }^{21}$ This has prompted reanalysis of the previously published LEED data. Addition of an ordered array of indium ad-atoms to the bulk-cut structure leads to a small but significant improvement in the Pendry R-factor. Density functional theory (DFT) calculations confirm that the occupation of three-fold hollow sites by the ad-atoms used in the LEED analysis is energetically feasible. As a result, simple electron counting dictates that each additional In atom (whose electron configuration is $5 s^{2} 5 p^{1}$ ) must contribute three electrons to the slab. It emerges that two of these electrons are trapped on the In ad-atoms in a directional $\operatorname{In}(\mathrm{I})$ lone pair surface state, while the third electron gives rise to an electron accumulation layer that has been characterised previously by both angle-integrated ${ }^{22,23}$ and angle-resolved photoemission (ARPES) measurements. ${ }^{24}$ Thus both the states in the bandgap and the electrons in the accumulation layer are associated with indium ad-atoms. This scenario differs from that proposed on the basis of low-energy ARPES measurements, where the accumulation layer was associated with surface oxygen vacancies and the bandgap state with bulk oxygen vacancies. ${ }^{24}$

\section{Results and discussion}

Valence band photoemission spectra of vacuum annealed $\operatorname{In}_{2} \mathrm{O}_{3}(111)(1 \times 1)$ excited at 600 $\mathrm{eV}$ and $6054 \mathrm{eV}$ photon energy are shown in the left panels of Figure 1. The most obvious change in the spectra upon increasing the photon energy is the pronounced enhancement of the relative intensity of the peak at the bottom of the valence band compared to other features in the spectra. At the same time, a weak peak near the Fermi level shows more muted intensity enhancement relative to the top of the valence band. Finally, a broad shoul- 
der toward the bottom of the bulk bandgap (identified as a gap state and marked as GS) in the Figure decreases in intensity relative to both the Fermi level peak and the valence band maximum. Both the gap state and the Fermi level peak are strongly attenuated for a sample that has been annealed in oxygen $\left(10^{-6}\right.$ mbar for 30 minutes at $\left.600{ }^{\circ} \mathrm{C}\right)$, as shown in the right hand panels of Figure 1, suggesting that they are inter-connected.
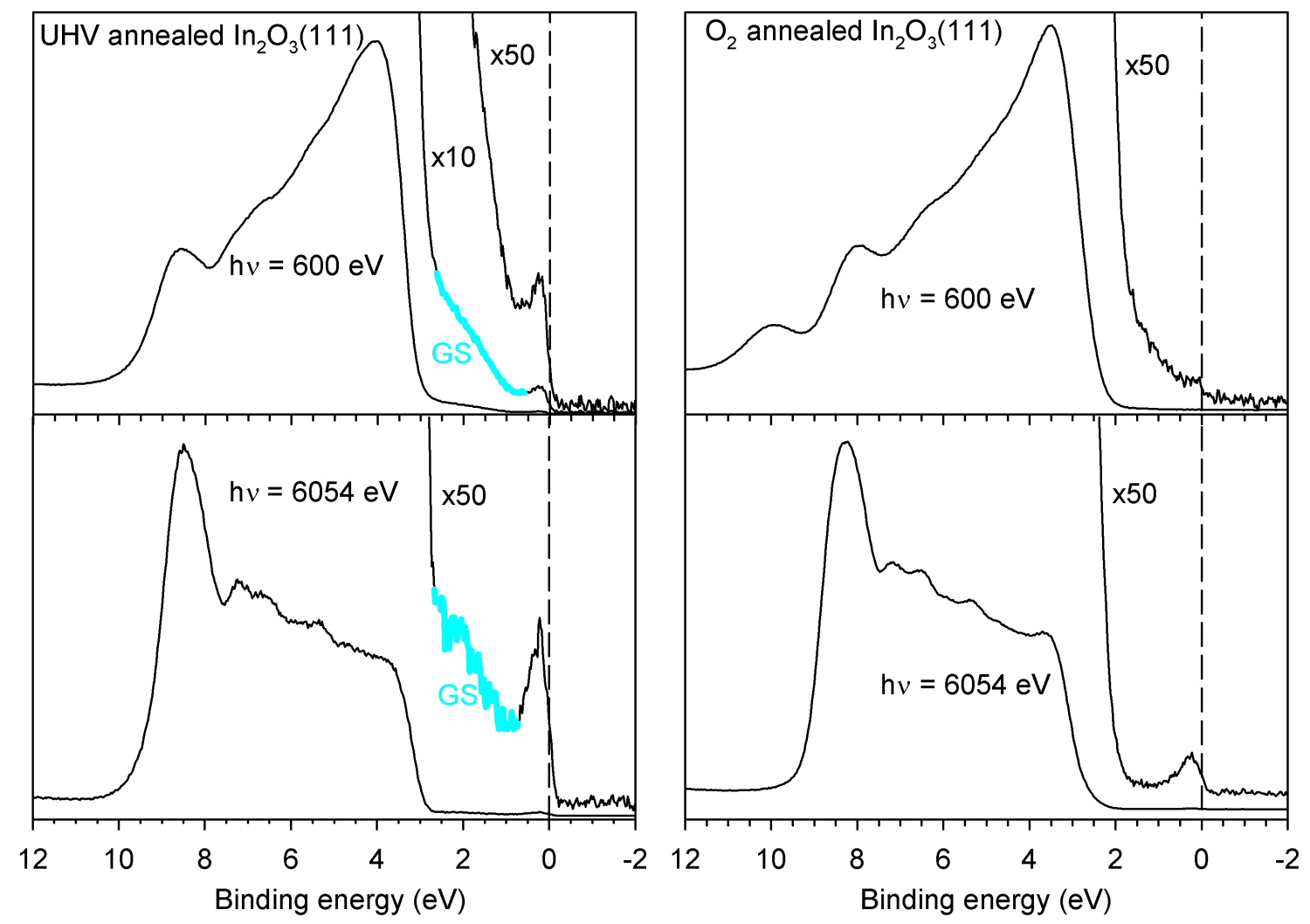

Figure 1: Hard and soft X-ray photoemission spectra of $\operatorname{In}_{2} \mathrm{O}_{3}(111)$ after annealing in UHV (left panels) and after annealing in oxygen (right panels). The gap state (GS) that is the main focus of this letter in highlighted in cyan in the left hand panels. Note the factor of 5 difference in the expansions of the highlighted region for the data taken at the two different photon energies (10 at $600 \mathrm{eV}$ and 50 at $6054 \mathrm{eV}$ ). The Fermi level is located at $0 \mathrm{eV}$ (dashed vertical line).

In principle, two factors need to be considered to understand these changes. Firstly, the electron inelastic mean free path increases with electron kinetic energy in the regime of our 
experiments from around $12 \AA$ at $600 \mathrm{eV}$ to $76 \AA$ at $6054 \mathrm{eV},{ }^{25}$ so structure associated with states localised at or near the surface will be stronger at the lower photon energy, in the absence of other influences. Secondly, intensities are dependent on ionisation cross sections and the atomic make-up of the electronic states involved. In particular, the cross-section for ionisation of In $5 s$ and In $5 p$ states decrease with increasing photon energy much less rapidly than for $\mathrm{O} 2 p$ states owing to the oscillatory behaviour of the radial wavefunction close to the In nucleus. ${ }^{26,27}$

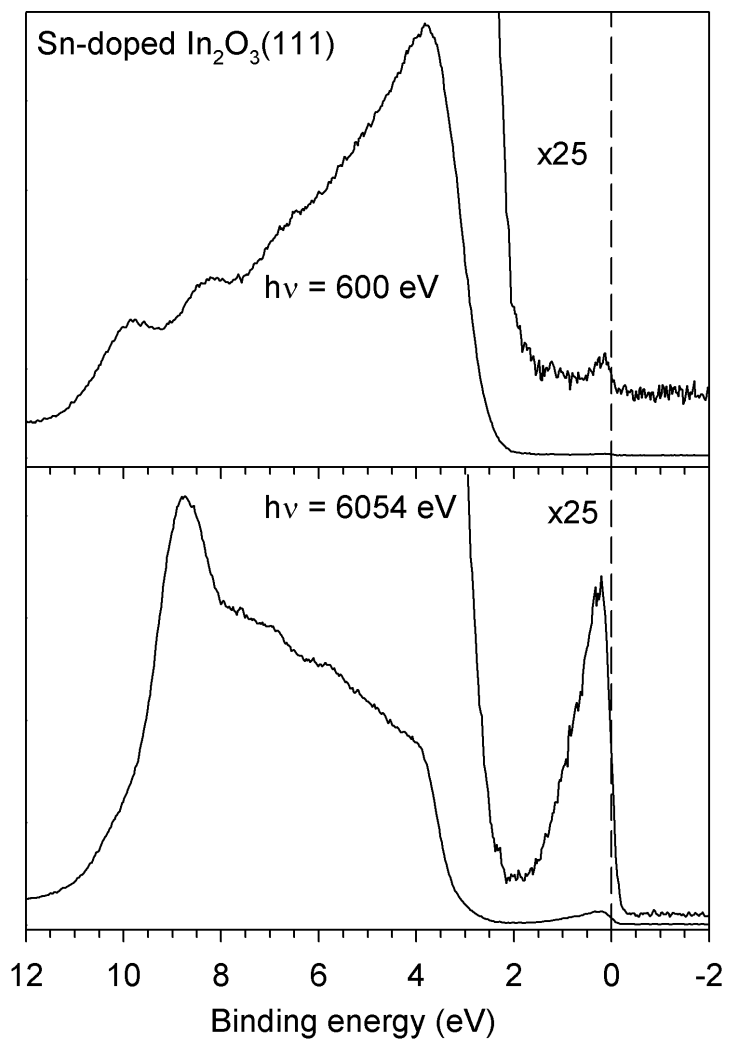

Figure 2: Hard and soft X-ray photoemission spectra of Sn-doped $\operatorname{In}_{2} \mathrm{O}_{3}(111)$. The increase in relative intensity of peaks at the bottom of the valence band and close to the Fermi energy provide a qualitative indication of the influence of cross-section effects.

Qualitatively, it is possible to establish the influence of cross-section effects by reference to photoemission data for Sn-doped $\operatorname{In}_{2} \mathrm{O}_{3}(111)$ shown in Figure 2. The carrier density in this sample is estimated to be about $4.5 \times 10^{20} \mathrm{~cm}^{-3}$ from the energy of the plasmon satellite 
observed on the In $3 d_{5 / 2}$ core line (Supplementary Figure S1). ${ }^{28}$ At this high carrier density the Fermi level lies above the charge neutrality level and there is carrier depletion rather than carrier accumulation at the surface. ${ }^{23}$ However, the Thomas-Fermi screening length is about $6.8 \AA$, so any band bending in the near-surface region must take place over this length range, which is less than the effective probing depth (which is usually considered to be three times the inelastic mean free path) for both the soft and hard X-rays used in the current study, as shown schematically in Figure 3. Thus XPS at both energies is dominated by the region where the electron gas is effectively homogeneous (even at $600 \mathrm{eV}$ only $40 \%$ of the photoemission intensity comes from the space charge region). The spectra in Figure 2 reveal an increase in intensity of the peak at the bottom of the valence band (as in Figure 1) and a related pronounced increase in the intensity of the conduction band peak near to the Fermi level. States at the bottom of the valence band and states in the conduction band both have pronounced In $5 s$ character and the intensity enhancement relative to states in upper part of the valence band reflects this high In $5 s$ contribution.

Comparing Figures 1 and 2 it is apparent that the increase in the relative intensity with photon energy of the conduction band feature is much less pronounced for the UHV annealed sample of $\mathrm{In}_{2} \mathrm{O}_{3}$ than for Sn-doped $\mathrm{In}_{2} \mathrm{O}_{3}$. This is because in the former case the conduction band peak derives from electrons in an accumulation whose overall thickness is of order $80 \AA .{ }^{24}$ Thus spectra excited at $600 \mathrm{eV}$ photon energy are completely dominated by the space charge region, whereas at $6054 \mathrm{eV}$ the effective sampling depth in the experiment extends through the space charge layer into regions where the carrier density is much lower, as shown in the schematic diagram of Figure 3. Thus, the effects of cross-section changes are offset by the increase in sampling depth. At the same time, the intensity of the structure in the gap decreases dramatically relative to that of both the conduction band and the top of the valence band on increasing the photon energy from $600 \mathrm{eV}$ to $6054 \mathrm{eV}$. Cross-section changes alone for a $5 s-5 p$ lone pair state would lead to an enhancement of the intensity relative to the top of the valence band, as is found in hard X-ray photoemission 


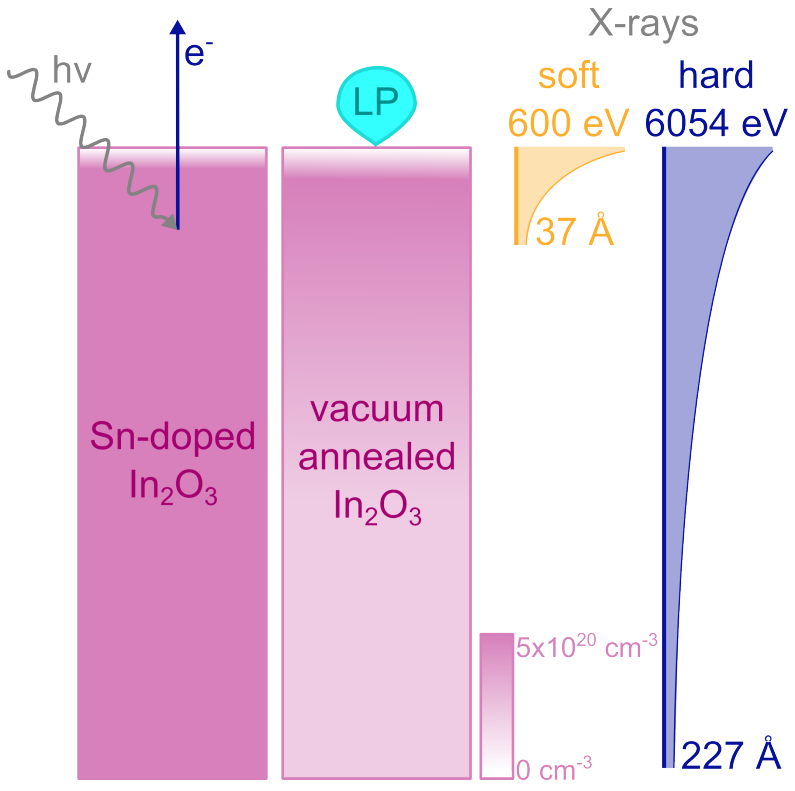

Figure 3: Schematic representation of the change in electron probing depth on increasing the photon energy from $600 \mathrm{eV}$ to $6054 \mathrm{eV}$ in relation to electron density in the electron accumulation layer (indicated by graded shading) formed on vacuum annealed $\operatorname{In}_{2} \mathrm{O}_{3}(111)$ $(1 \times 1)$. Electron density in the surface lone pair $(\mathrm{LP})$ is highlighted in cyan. The essentially homogenous electron density in Sn-doped $\mathrm{In}_{2} \mathrm{O}_{3}$ is also shown in a schematic. The exponential decrease in the contribution to the photoemission signal with increasing depth is indicated in the two curves on the right of the figure, which extend over a distance of three times the inelastic mean-free path. Note that in the semi-classical infinite barrier model used to deal with band bending, ${ }^{22-24}$ the electron density goes to zero at the surface itself due to the potential barrier. 
of the lone pair state in $\mathrm{SnO} \cdot{ }^{29,30}$ However for lone pair states localised entirely on surface cations, cross-section effects will again be offset by changes in the effective sampling depth, to an extent that should be even more pronounced than when dealing with electrons in the accumulation layer. This explains the decrease in intensity of the gap state feature relative to the conduction band feature on increasing the photon energy. In summary, the left hand panel of Figure 1 alone tells us already that the GS is much more confined in depth to the surface than the spread of the electrons in the accumulation layer. Comparing the left hand panel off Figure 1 with Figure 2, we further learn that the overall depth distribution of the accumulation layer is shallower than the effective probing depth for $6 \mathrm{keV}$ electrons (over 200 $\AA)$. Thus $200 \AA>t$ (accumulation layer) $>t($ GS), where the $t$ are effective thicknesses. This scenario differs from that proposed previously based on ARPES data ${ }^{24}$ and the assumption that surface oxygen vacancies are shallow donors and bulk oxygen vacancies are deep states. ${ }^{11}$

To provide atomistic insights into the nature of the lone pair surface states, we turn to first-principles calculations. Mapping of the potential energy surface for In adatoms on the surface of $\operatorname{In}_{2} \mathrm{O}_{3}$ reveals a number of threefold oxygen hollow sites as local energy minima, as shown Figure 4. More specifically we may distinguish between A sites where the three-fold oxygen hollow surrounds an oxygen atom at the bottom of the outer quadrupolar layer, and B sites where this sub-surface oxygen is missing - the sub-surface sites would be occupied by an anion for (111) surfaces of materials with the stoichiometric $\mathrm{MO}_{2}$ fluorite structure. In analysing the LEED data, we were constrained to dealing with sites with local $\mathrm{C}_{3}$ symmetry i.e. all the oxygen atoms were required to have the same $\mathrm{z}$ coordinate. Only one of the B sites met this requirement and the subsequent discussion is based on occupation of this B site by the ad-atom. However, it must be noted that the adsorption minima are somewhat deeper for the A sites $(-0.45 \mathrm{eV})$ than the B sites $(-0.32 \mathrm{eV})$ in the DFT calculations.

In the LEED analysis, three equal In-O bond lengths of $2.15 \AA$ were found for the ad- 

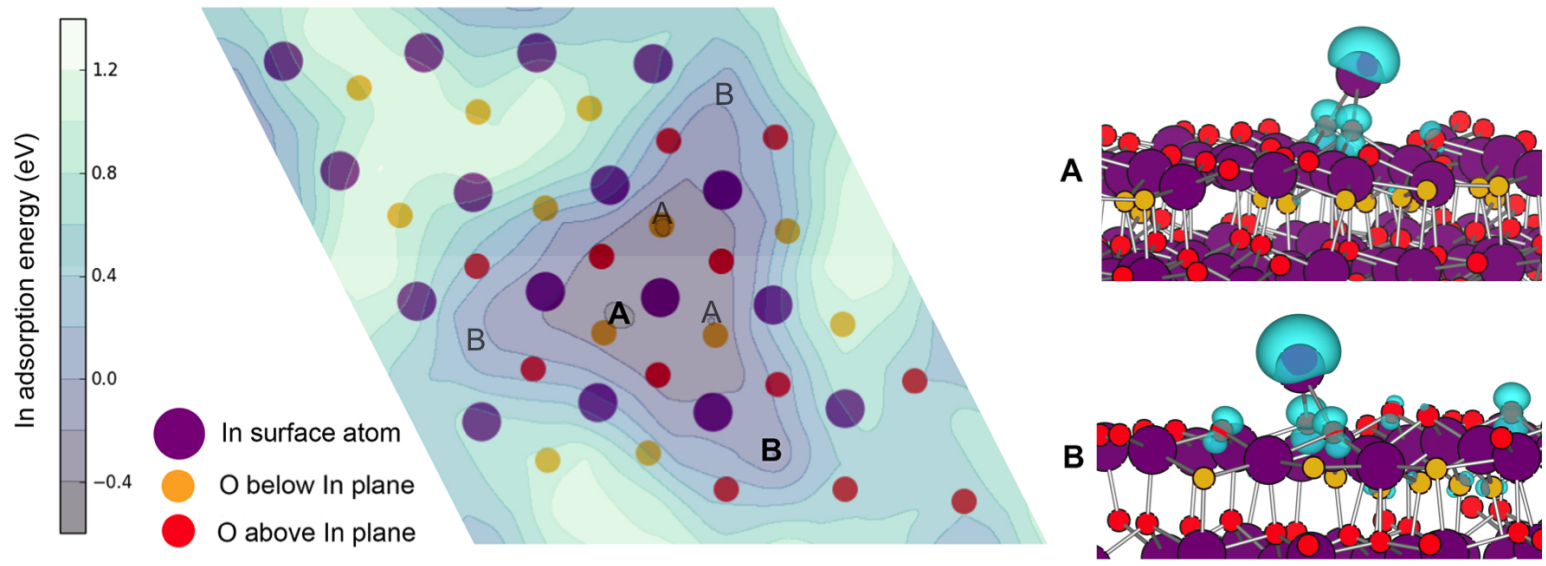

Figure 4: Calculated potential energy surface of single In ad-atom incorporation onto the (111) termination of $\operatorname{In}_{2} \mathrm{O}_{3}$. The adsorption energy is for the process: $\mathrm{In}(\mathrm{s})+$ bare-surface In-on-surface. A number of local minima are found with total energy within several meV of each other. Also plotted is the electron density associated with the sub bandgap feature observed above the valence band in the electronic density of states for surface sites labeled A and B: it is localised asymmetric electron density characteristic of an $\operatorname{In}(\mathrm{I})$ lone pair.

atoms. In the DFT calculations, the allowance of lateral relaxation led to the ad-atom moving off-centre in the hollow site to give three different and slightly longer In-O bond lengths of $2.28 \AA, 2.39 \AA$ and $2.89 \AA$. The In-O bond length emerging from the LEED analysis is within the range between $2.13 \AA$ and $2.23 \AA$ found in the bulk crystal structure of $\operatorname{In}_{2} \mathrm{O}_{3}$. ${ }^{31}$ Some of the discrepancy between LEED and DFT may arise from partial compensation the surface In(I) donors by near-surface oxygen interstitials, so that some of the ad-atoms are $\operatorname{In}(\mathrm{III})$ rather than $\operatorname{In}(\mathrm{I})$. Given a bulk lattice parameter of $10.117 \AA$, the presence of an $\operatorname{In}(\mathrm{I})$ oneelectron donor within each surface unit cell would lead to a $2 \mathrm{D}$ electron density of $5.6 \times 10^{13}$ $\mathrm{cm}^{-2}$ within the electron accumulation layer. The value inferred from measurements of the wavevector at the Fermi energy in angle resolved photoelectron spectra is $4.2 \times 10^{13} \mathrm{~cm}^{-2},{ }^{24}$ thus suggesting $25 \%$ of the donor centres are compensated. This would lead to reduction in the average In-O bond length. However a more extended set of experimental LEED data, including measurements at non-normal incidence, are needed to further explore details of the surface structure. The partial densities of states for a bare slab and for a slab with a single uncompensated indium ad-atom per surface unit cell in a site B are shown in Figure 5. The 
In ad-atoms result in two changes to the electronic structure: (i) one electron is dispersed into the delocalised conduction band of $\operatorname{In}_{2} \mathrm{O}_{3}$ resulting in n-type doping and thereby the two-dimensional gas in the near surface region; ${ }^{22-24}$ (ii) two electrons remain trapped in a state localised on the indium ad-atom. This state sits just above the main valence band edge and extends into the bulk bandgap. Inspection of the partial electronic densities of states shows that the gap state has pronounced In $5 s$ and In $5 p$ character, as expected for a directional lone pair, but with additional mixing with $\mathrm{O} 2 p$ states as required by recent models for development of lone pairs. ${ }^{9,10}$

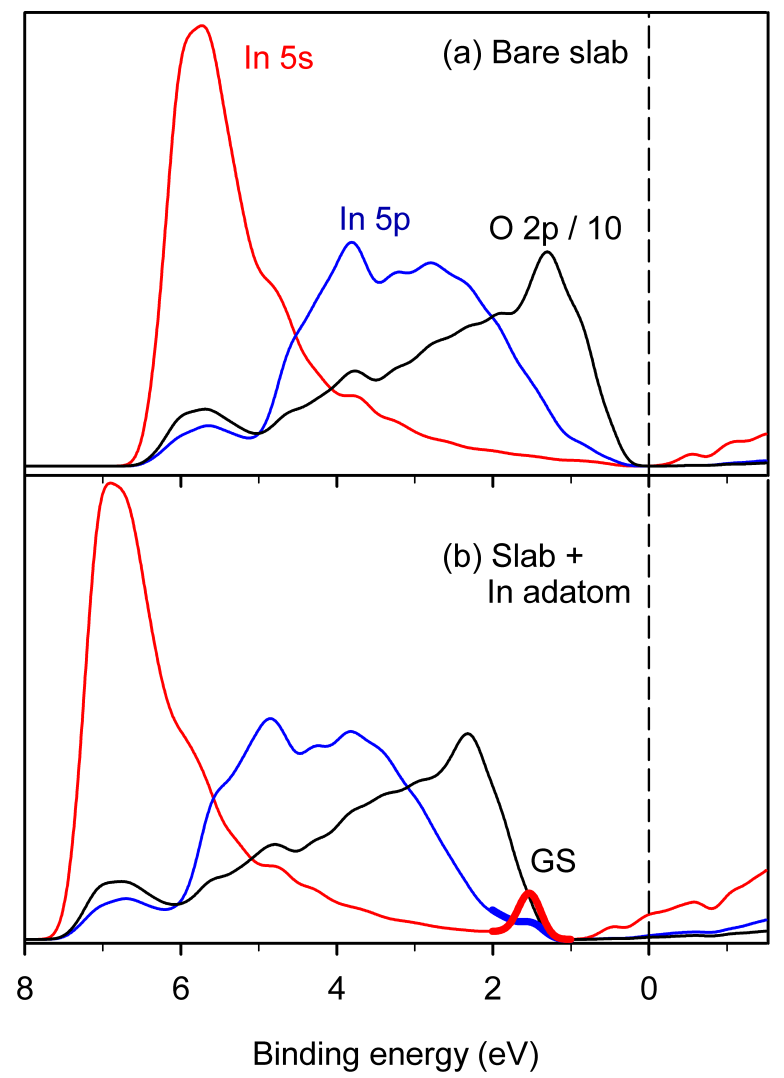

Figure 5: O $2 p$, In $5 s$ and In $5 p$ partial densities of states (PDOS) for $\operatorname{In}_{2} \mathrm{O}_{3}(111)$ : (a) bare (11) slab (b) after inclusion of a single In adatom in each surface unit cell in site B. Binding energies are given relative to the Fermi level, which is indicated by a dashed line. The lower panel reveals the appearance of a new gap state (GS) just above the valence band maximum with mixed In $5 s-5 p$ (identified in bold) and $\mathrm{O} 2 p$ character, as well as a shift in the Fermi level from mid-gap into the conduction band. Note that the O $2 p$ PDOS has been divided by 10 to allow visual comparison with In $5 s$ and $5 p$ states. 
In the simple chemical nomenclature of the ionic model, the adatoms are $\mathrm{N}-2$ valent $\mathrm{In}^{+}$ ions, in contrast to $\mathrm{In}^{3+}$ formally present in the bulk. However, due to pronounced covalency these different charge states are better represented as $\operatorname{In}(\mathrm{I})$ and $\operatorname{In}(\mathrm{III})$ respectively. Although the $\operatorname{In}(\mathrm{I})$ oxide $\operatorname{In}_{2} \mathrm{O}$ is not known as a bulk solid phase, $\operatorname{In}_{2} \mathrm{O}$ has been characterised in the gas phase. ${ }^{32}$ A limited number of solid oxide phases containing $\operatorname{In}(\mathrm{I})$ are also known, including the tungsten bronze $\operatorname{In}_{\mathrm{x}} \mathrm{WO}_{3}{ }^{33}$ and $\mathrm{In}(\mathrm{I})-\beta^{\prime \prime}$-alumina. ${ }^{34} \mathrm{In}_{2} \mathrm{O}$ has also been deposited onto $\operatorname{InAs}(001)$ surfaces, where it was characterised by STM. ${ }^{35}$ Thus the formation of surface In(I) sites is broadly consistent with the known chemistry of indium. Lone pair states of the sort we have identified are expected to have a profound impact on the catalytic ${ }^{36}$ and photocatalytic activity of $\operatorname{In}_{2} \mathrm{O}_{3} \cdot{ }^{13}$ In particular directional lone pairs will confer Lewis basicity on the surface indium sites, and at the same time the surface lone pairs, which lie just above the top of the bulk valence band, will act as centres for trapping photogenerated holes. Holes located at the surface are optimally placed to bring about oxidation reactions, such as the destruction of organic pollutants. ${ }^{37}$

The combination of hard and soft X-ray photoemission measurements performed in tandem with first principles density functional theory calculations have conclusively established that electronic states found within the bandgap of $\operatorname{In}_{2} \mathrm{O}_{3}(111)$ arise from electron lone pairs localised on surface indium ad-atoms. These ad-atoms also provide the electrons for the well-known electron accumulation layer that develops on the surface of $\operatorname{In}_{2} \mathrm{O}_{3}$ under reducing conditions. ${ }^{23,24}$ The current interpretation differs from that advanced previously, where the gap state was presumed to arise from bulk oxygen vacancies and the electrons in the accumulation layer from surface oxygen vacancies. ${ }^{24}$ This explanation is not consistent with hard and soft XPS when considered together. The current picture is much simpler and is supported by LEED I/V data, which reveal the presence of the ad-atoms first proposed on the basis of STM measurements. It remains to be seen if the ideas developed here can be extended to other post transition metal oxides such as $\mathrm{SnO}_{2}{ }^{14,15}$ and $\mathrm{Ga}_{2} \mathrm{O}_{3}{ }^{16}$ where in-gap 
states are also known to develop under reducing conditions.

\section{Experimental}

Undoped $\mathrm{In}_{2} \mathrm{O}_{3}$ and $3 \%$ Sn-doped $\mathrm{In}_{2} \mathrm{O}_{3}$ (ITO) single crystal thin films with atomically flat surfaces were grown on $\mathrm{Y}-\mathrm{ZrO}_{2}(111)$ substrates in an oxide molecular beam epitaxy system at a substrate temperature of $700{ }^{\circ} \mathrm{C}$ with an oxygen plasma pressure of $3 \times 10^{-5}$ mbar to a thickness of $210 \mathrm{~nm} .{ }^{38}$ The initial carrier concentrations for the as-grown $\mathrm{In}_{2} \mathrm{O}_{3}$ and ITO films were $2 \times 10^{18} \mathrm{~cm}^{-3}$ and $4 \times 10^{20} \mathrm{~cm}^{-3}$, respectively, as determined by Hall measurements and further confirmed by infrared reflectivity. The carrier density increased to a higher value of around $1.5 \times 10^{19} \mathrm{~cm}^{-3}$ for the undoped sample after the experimental measurements in UHV, a value above the limit for the onset of degeneracy. For the Sn-doped sample it was possible to estimate an in situ carrier density of around $4.5 \times 10^{20} \mathrm{~cm}^{-3}$ from measurement of a plasmon satellite on the In $3 d$ core line at $0.64 \mathrm{eV}$ (Supplementary Figure S1). ${ }^{28}$

Photoemission spectra were obtained at the I09 beamline of the Diamond Light Source, UK. This beamline delivers photons with energies ranging from $100 \mathrm{eV}$ to $18000 \mathrm{eV}$ using two canted undulators, providing both soft and hard X-rays focused to the same point on the sample. Hard X-rays $(\mathrm{h} \nu=6054 \mathrm{eV})$ were selected using a cooled $\mathrm{Si}(111)$ double crystal monochromator coupled with an additional $\mathrm{Si}(004)$ channel-cut high resolution monochromator. Soft X-rays $(\mathrm{h} \nu=600 \mathrm{eV})$ were obtained using a plane grating monochromator (300 lines/mm). The end station is equipped with a VG Scienta EW4000 electron analyzer with $\pm 30^{\circ}$ angular acceptance. The photon beam is perpendicular to the electron emission direction. The photon beam is polarized in the plane of the orbit (horizontally) resulting in the electric vector being aligned with the electron emission direction. The sample was placed in a grazing incidence geometry $\left(<5^{\circ}\right)$, with the surface normal in the plane defined 
by the photon beam and electron emission direction (p-polarized), thereby significantly enhancing the count rate. The experiments were performed at room temperature. The total experimental energy resolution was better than $0.3 \mathrm{eV}$ for all photon energies. Experiments were conducted on an undoped sample which had been previously annealed in oxygen ${ }^{24}$ and on the same sample after gentle Ar ion bombardment (6 mA, $500 \mathrm{eV}$ for 5 minutes) followed by annealing at $500 \mathrm{C}$ for 30 minutes in UHV. The UHV annealed sample gave a well-defined $(1 \times 1)$ LEED pattern. Another $\operatorname{In}_{2} \mathrm{O}_{3}$ thin film prepared with very similar conditions in a different UHV chamber connected directly with STM, shows the appearance of ordered bright dots (as shown in Supplementary Figure S2) characteristic of the In ad-atoms reported by M. Wagner et al. ${ }^{19}$ The as-presented Sn-doped sample was studied for comparison.

First-principles calculations were performed on the pristine (111) surface of $\operatorname{In}_{2} \mathrm{O}_{3}$, which is the lowest energy termination of the crystal and contains planes of charge neutral $\left\{\mathrm{O}_{12} \operatorname{In}_{16} \mathrm{O}_{12}\right\}$ trilayers. A slab model of 4 quadrupolar trilayers (160 atoms) was used, as previously reported. ${ }^{39}$ The potential energy surface for indium adatom adsorption was assessed by introducing a $10 \times 10$ grid on the (111) surface and calculating the total energy for a locally relaxed structure at each grid point. The total energy and electronic structure were calculated within DFT as implemented in the VASP code. The electronic wavefunctions were represented using planes waves with a kinetic energy cutoff of $500 \mathrm{eV}$, and the Brillouin zone was sampled using a k-point grid of $2 \times 2 \times 1$ ( $4 \times 4 \times 1$ for the density of states). All calculations were performed using the PBEsol exchange-correlation functional.

The dynamical LEED calculations were performed using the Tensor-LEED program. ${ }^{40}$ The relativistic phase shifts were calculated using the phase shift program ${ }^{41}$ that is packaged with Tensor-LEED. The agreement between the theory and the experiment was tested using the Pendry R-factor and the error bars quoted are calculated using the Pendry RRfunction. ${ }^{42}$ Four different sets of phase shifts were used: two for oxygen and two for indium. 
At the beginning of the analysis the Debye temperatures were set to $108 \mathrm{~K}$ for In and 500 $\mathrm{K}$ for $\mathrm{O}$. The $l_{\max }$ value was set to 8 and the imaginary part of inner potential was set to $5.0 \mathrm{eV}$. These values were optimised in the final stages of the analysis to $100 \mathrm{~K}$ and $350 \mathrm{~K}$ for the surface and bulk In respectively; to $1300 \mathrm{~K}$ and $2500 \mathrm{~K}$ for the surface and bulk O; to 8 for $l_{\max }$ and to $5.5 \mathrm{eV}$ for imaginary part of inner potential. The real part of the inner potential is independent of energy and was allowed to relax as is normal in LEED analysis. Starting from a bulk cut structure, a single indium ad-atom was added to one of the threefold hollow sites identified in the calculations discussed above and relaxation was allowed in the outer two quadrupolar layers and for the In ad-atom. The geometrical parameters are optimized only in the vertical ( $\mathrm{z}$ ) direction: normal incidence LEED is not very sensitive to the lateral parameters and the published data set was limited in range. Although 81 atoms were therefore allowed to relax, symmetry constraints dictated that only 28 positional parameters were refined, along with the real part of the inner potential and the $\mathrm{z}$ coordinate of the ad-atom. This corresponds to $(1270 / 30) \mathrm{eV}=42 \mathrm{eV}$ of raw data per parameter. All parameters were refined simultaneously. For indium atoms the final error bars are between $0.02 \AA$ and $0.05 \AA$ in the outer quadrupolar layer and between $0.08 \AA$ and $0.12 \AA$ in the next layer. The error bars on the oxygen positions are larger and increase with depth below the surface from $0.06 \AA$ and $0.08 \AA$ in the outermost layer through ranges of $0.04 \AA-0.10 \AA$; $0.10 \AA-0.25 \AA$; and $0.12 \AA-0.16 \AA$ in the next three layers. The final Pendry R-factor was found to be 0.27 after inclusion of a single ad-atom per unit cell at one of B positions (see below) suggested by the DFT calculations, as compared with $\mathrm{R}=0.31$ without inclusion of ad-atoms. The results of the LEED analysis, including measured and simulated I/V profiles, are given as given as supplementary data. 


\section{Acknowledgement}

D.W.D. thanks the Engineering and Physical Sciences Research Council (EPSRC) for sup-

port via the Centre for Doctoral Training in Sustainable Chemical Technologies (EP/L016354/1).

Calculations were carried out on the Balena HPC cluster at the University of Bath, which is maintained by Bath University Computing Services. V.R.D. thanks the Engineering and Physical Sciences Research Council (EPSRC) for support (EP/NO15800/1). K.H.L.Z. gratefully acknowledges the funding support by Clarendon scholarships at university of Oxford and the Thousand Youth Talents Program at Xiamen University. K.P. thanks the Academy of Finland ( grant 277829) and the CSC - Finnish IT Center for Science.

\section{References}

(1) Orgel, L. Journal of the Chemical Society 1959, 0, 3815-3819.

(2) Dunitz, J.; Orgel, L. Advances in Inorganic Chemistry and Radiochemistry 1960, 2, $1-60$.

(3) Walsh, A.; Watson, G. W. Physical Review B - Condensed Matter and Materials Physics 2004, 70,1-7.

(4) Walsh, A.; Watson, G. W. Journal of Physical Chemistry B 2005, 109, 18868-18875.

(5) Walsh, A.; Watson, G. W. Journal of Solid State Chemistry 2005, 178, 1422-1428.

(6) Walsh, A.; Watson, G. W.; Payne, D. J.; Edgell, R. G.; Guo, J.; Glans, P. A.; Learmonth, T.; Smith, K. E. Physical Review B - Condensed Matter and Materials Physics 2006, 73, 1-13.

(7) Raulot, J. M.; Baldinozzi, G.; Seshadri, R.; Cortona, P. Solid State Sciences 2002, 4, $467-474$. 
(8) Waghmare, U. V.; Spaldin, N. A.; Kandpal, H. C.; Seshadri, R. Physical Review B Condensed Matter and Materials Physics 2003, 67, 10.

(9) Payne, D. J.; Egdell, R. G.; Walsh, A.; Watson, G. W.; Guo, J.; Glans, P. A.; Learmonth, T.; Smith, K. E. Physical Review Letters 2006, 96, 1-4.

(10) Walsh, A.; Payne, D. J.; Egdell, R. G.; Watson, G. W. Chemical Society Reviews 2011, 40, 4455-4463.

(11) Lany, S.; Zakutayev, A.; Mason, T. O.; Wager, J. F.; Poeppelmeier, K. R.; Perkins, J. D.; Berry, J. J.; Ginley, D. S.; Zunger, A. Physical Review Letters 2012, $108,2-6$.

(12) Buckeridge, J.; Catlow, C. R. A.; Farrow, M. R.; Logsdail, A. J.; Scanlon, D. O.; Keal, T. W.; Sherwood, P.; Woodley, S. M.; Sokol, A. A.; Walsh, A. Physical Review Materials 2018, 2, 054604 .

(13) Ghuman, K. K.; Hoch, L. B.; Szymanski, P.; Loh, J. Y. Y.; Kherani, N. P.; ElSayed, M. A.; Ozin, G. A.; Singh, C. V. Journal of the American Chemical Society 2016, 138, 1206-1214.

(14) Cox, P. A.; Egdell, R. G.; Harding, C.; Patterson, W. R.; Tavener, P. J. Surface Science 1982, 123, 179-203.

(15) Egdell, R. G.; Eriksen, S.; Flavell, W. R. Solid State Communications 1986, 60, 835838.

(16) Regoutz, A.; Egdell, R. G.; Morgan, D. J.; Palgrave, R. G.; Téllez, H.; Skinner, S. J.; Payne, D. J.; Watson, G. W.; Scanlon, D. O. Applied Surface Science 2015, 349, 970982.

(17) Panaccione, G.; Kobayashi, K. Surface Science 2012, 606, 125-129. 
(18) Pussi, K.; Matilainen, A.; Dhanak, V. R.; Walsh, A.; Egdell, R. G.; Zhang, K. H. L. Surface Science 2012, 606, 1-6.

(19) Wagner, M.; Seiler, S.; Meyer, B.; Boatner, L. A.; Schmid, M.; Diebold, U. Advanced Materials Interfaces 2014, 1, 1-6.

(20) Wagner, M.; Lackner, P.; Seiler, S.; Gerhold, S.; Osiecki, J.; Schulte, K.; Boatner, L. A.; Schmid, M.; Meyer, B.; Diebold, U. Physical Review Letters 2016, 117, 1-6.

(21) Zhang, K. H.; Payne, D. J.; Palgrave, R. G.; Lazarov, V. K.; Chen, W.; Wee, A. T.; McConville, C. F.; King, P. D.; Veal, T. D.; Panaccione, G.; Lacovig, P.; Egdell, R. G. Chemistry of Materials 2009, 21, 4353-4355.

(22) King, P. D.; Veal, T. D.; Payne, D. J.; Bourlange, A.; Egdell, R. G.; McConville, C. F. Physical Review Letters 2008, 101, 2-5.

(23) King, P. D. C.; Veal, T. D.; Fuchs, F.; Wang, C. Y.; Payne, D. J.; Bourlange, A.; Zhang, H.; Bell, G. R.; Cimalla, V.; Ambacher, O.; Egdell, R. G.; Bechstedt, F.; McConville, C. F. Physical Review B - Condensed Matter and Materials Physics 2009, $79,1-10$.

(24) Zhang, K. H.; Egdell, R. G.; Offi, F.; Iacobucci, S.; Petaccia, L.; Gorovikov, S.; King, P. D. Physical Review Letters 2013, 110, 1-5.

(25) Tanuma, S.; Powell, C.; Penn, D. Surface and Interface Analysis 1994, 21, 165-176.

(26) Walsh, A.; Da Silva, J. L.; Wei, S. H.; Körber, C.; Klein, A.; Piper, L. F.; Demasi, A.; Smith, K. E.; Panaccione, G.; Torelli, P.; Payne, D. J.; Bourlange, A.; Egdell, R. G. Physical Review Letters 2008, 100, 2-5.

(27) Körber, C.; Krishnakumar, V.; Klein, A.; Panaccione, G.; Torelli, P.; Walsh, A.; Da Silva, J. L. F.; Wei, S.-H.; Egdell, R. G.; Payne, D. J. Physical Review B 2010, 81, 165207. 
(28) Bourlange, A.; Payne, D. J.; Palgrave, R. G.; Zhang, H.; Foord, J. S.; Egdell, R. G.; Jacobs, R. M. J.; Veal, T. D.; King, P. D. C.; McConville, C. F. Journal of Applied Physics 2009, 106, 013703.

(29) Ogo, Y.; Hiramatsu, H.; Nomura, K.; Yanagi, H.; Kamiya, T.; Kimura, M.; Hirano, M.; Hosono, H. Physica Status Solidi (A) Applications and Materials Science 2009, 206, $2187-2191$.

(30) Quackenbush, N. F.; Allen, J. P.; Scanlon, D. O.; Sallis, S.; Hewlett, J. A.; Nandur, A. S.; Chen, B.; Smith, K. E.; Weiland, C.; Fischer, D. A.; Woicik, J. C.; White, B. E.; Watson, G. W.; Piper, L. F. Chemistry of Materials 2013, 25, 3114-3123.

(31) Marezio, M. Acta Crystallographica 1966, 20, 723-728.

(32) Lakin, N. M.; van den Hoek, G.; Beattie, I. R.; Brown, J. M. Journal of Chemical Physics 1997, 107, 4439.

(33) Ekstrom, T.; Parmentier, M.; Watts, K. A.; Tilley, R. J. D. Journal of Solid State Chemistry 1984, 54, 365-377.

(34) Cetinkol, M.; Lee, P. L.; Wilkinson, A. P. Materials Research Bulletin 2007, 42, 713719 .

(35) Shen, J.; Melitz, W.; Feldwinn, D. L.; Lee, S.; Droopad, R.; Kummel, A. C. In Physics and Technology of High-K Materials 8; Kar, S., VanElshocht, S., Misra, D., Houssa, M., Landheer, D., Kita, K., Eds.; 2010; pp 105-116.

(36) Martin, O.; Martín, A. J.; Mondelli, C.; Mitchell, S.; Segawa, T. F.; Hauert, R.; Drouilly, C.; Curulla-Ferré, D.; Pérez-Ramírez, J. Angewandte Chemie International Edition 2016, 55, 6261-6265.

(37) Oropeza, F. E.; Davies, B.; Palgrave, R. G.; Egdell, R. G. Physical Chemistry Chemical Physics 2011, 13, 7882-7891. 
(38) Zhang, K. H. L.; Lazarov, V. K.; Veal, T. D.; Oropeza, F. E.; McConville, C. F.; Egdell, R. G.; Walsh, A. Journal of Physics: Condensed Matter 2011, 23, 334211.

(39) Walsh, A.; Catlow, C. R. A. Journal of Materials Chemistry 2010, 20, 10438-10444.

(40) Van Hove, M.; Moritz, W.; Over, H.; Rous, P.; Wander, A.; Barbieri, A.; Materer, N.; Starke, U.; Somorjai, G. Surface Science Reports 1993, 19, 191-229.

(41) Barbieri, A.; Vanhove, M. A. unpublished

(42) Pendry, J. B. Journal of Physics C: Solid State Physics 1980, 13, 937-944. 